\title{
Effects after starting or switching from bisphosphonate to romosozumab or denosumab in Japanese postmenopausal patients
}

\author{
Tomohiro Shimizu ${ }^{1}(1) \cdot$ Kosuke Arita $^{1} \cdot$ Eihiro Murota $^{2} \cdot$ Shigeto Hiratsuka $^{1,2} \cdot$ Ryo Fujita $^{1,3} \cdot$ Hotaka Ishizu $^{1}$. \\ Tsuyoshi Asano ${ }^{1} \cdot$ Daisuke Takahashi $^{1} \cdot$ Masahiko Takahata $^{1} \cdot$ Norimasa Iwasaki $^{1}$
}

Received: 3 February 2021 / Accepted: 23 March 2021 / Published online: 13 April 2021

(c) The Japanese Society Bone and Mineral Research 2021

\begin{abstract}
Purpose We aimed to investigate the longitudinal changes in bone metabolic markers and bone mineral density (BMD) after starting or switching from bisphosphonate (BP) to romosozumab (ROMO) or denosumab (DENO) therapies over 12 months and to determine predictors that establish associations with changes in BMD among the patients received the ROMO therapy. Methods Postmenopausal osteoporosis patients with a high risk of fracture-154 in total—were recruited; their therapies were switched to ROMO or DENO from BP/naïve or vitamin D (ND) (ND-ROMO: 43, BP-ROMO: 38, ND-DENO: 38, and BP-DENO: 35). Longitudinal changes in bone metabolic markers and BMD were evaluated.

Results ROMO groups showed significant increases in BMD of the lumbar spine at 6 and 12 months and femoral neck at 12 months compared to the DENO groups. Although BP-ROMO showed significant increase in the lumbar spine BMD compared to BP-DENO, there were no significant differences in femoral neck and total hip BMDs between BP-ROMO and BP-DENO. Among the ROMO groups, \% changes of BMD from baseline to 12 months were associated with bone metabolic markers at baseline and changes in TRACP-5b from baseline to 3 months.

Conclusions ROMO continuously increased BMD for 12 months and performed better than DENO. On the other hand, effects of ROMO switched from BP on BMD of femoral neck and total hip were almost same with DENO. Bone metabolic markers at baseline and changes in TRACP-5b from baseline to 3 months may predict the efficacy of ROMO after 12 months of administration.
\end{abstract}

Keywords Postmenopausal osteoporosis $\cdot$ Romosozumab $\cdot$ Denosumab $\cdot$ Bone metabolic marker

\section{Introduction}

Osteoporosis is a chronic, progressive condition that requires long-term management. An estimated 9 million new osteoporosis-related fractures were reported worldwide in the

Tomohiro Shimizu and Kosuke Arita contributed equally to this manuscript.

Tomohiro Shimizu

simitom@wg8.so-net.ne.jp

1 Department of Orthopaedic Surgery, Faculty of Medicine and Graduate School of Medicine, Hokkaido University, Kita-15 Nishi-7, Kita-ku, Sapporo, Hokkaido 060-8638, Japan

2 Department of Orthopaedic Surgery, Wajyokai Sapporo Hospital, Sapporo, Japan

3 Department of Orthopaedic Surgery, Hokkaido Orthopaedic Memorial Hospital, Sapporo, Japan year 2000 [1], and 75 million people in the United States, Europe, and Japan are affected by osteoporosis [2]. In 2010 there were estimated to be 158 million individuals at high fracture risk. Demographic shifts mean that this figure is likely to double by 2040 [3]. Patients with osteoporosis are usually treated with bisphosphonates (BP) as first-line therapy because of their high effectiveness, long-term benefits, and price considerations. In patients who are at high risk of fractures and patients who do not respond sufficiently, such as those who have a persistent low bone mineral density (BMD) or those who develop fractures regardless of BP therapy, starting or switching to potent therapies is the common clinical practice.

Romosozumab (ROMO) is a monoclonal antibody that binds to and inhibits sclerostin. ROMO therapy has a dual effect; it increases bone formation and decreases bone resorption $[4,5]$. Because of this unique dual effect, the anabolic window, which determines the effects of osteoporosis 
treatment, is assumed to be larger for ROMO than for other osteoporosis treatments [6]. Large-cohort randomized control studies showed that ROMO was associated with increased bone mineral density and formation and decreased bone resorption and lower risk of vertebral fractures than the placebo at 12 months [5, 7]. Furthermore, other randomized cohort studies showed that ROMO treatment for 12 months followed by alendronate treatment significantly lowered the risk of fractures than treatment with alendronate and teriparatide $[8,9]$. Contrarily, there is limited information comparing ROMO with denosumab (DENO), a human monoclonal antibody that targets the osteoclast differentiation factor/receptor activator of the NF-kB ligand [10].

Japan approved ROMO in March 2019 and was the first to do so. Recent clinical reports on Japanese patients with postmenopausal osteoporosis in the real-world setting also showed that ROMO was effective in increasing BMD and preventing fractures even after 4-6 months of treatment $[11,12]$. Although these reports showed that differences in prior treatment and early changes in bone turnover markers were associated with changes in BMD, there was still a paucity of information regarding the efficacy of ROMO until 12 months and its predictors in the real-world setting. Therefore, the current study aimed to investigate the longitudinal changes in bone metabolic markers and bone mineral density (BMD) after starting or switching from BP to ROMO or DENO therapies over 12 months and to determine predictors that establish associations with changes in BMD among the patients received the ROMO therapy. The hypotheses of this current study were that ROMO therapy could be one of most ideal treatment options for postmenopausal osteoporosis patients with high-risk fragility fracture and that bone metabolic markers before administration could be predictor for the therapeutic efficacy of ROMO.

\section{Materials and methods}

\section{Study design and subjects}

This study was conducted in accordance with the ethical standards of the Declaration of Helsinki and approved by the Hokkaido University Hospital Institutional Review Board (\#020-0188). A total of 327 patients with postmenopausal osteoporosis met the criteria defined by the Japanese guidelines for prevention and treatment of osteoporosis [13, 14], who were at a high risk of fractures, were visited to our clinical team from January 2014 to December 2019. They were treated using DENO (60 mg, subcutaneously every 6 months), zoledronic acid (Zol) $(5 \mathrm{mg}$, intravenous drip infusion), and ROMO (210 mg, subcutaneously every month) in combination with a daily oral active vitamin D by our clinical team from January 2014 to December 2019
(Fig. 1). A high risk of fracture was defined by (1) BMD < -2.5 of SD (standard deviation) and one or more fragility fractures, (2) lumbar BMD $<-3.3$ of SD [15, 16], (3) two or more fragility fractures $[15,17]$, or (4) semi-quantitative evaluation [18] of existing grade 3 vertebral fracture [17]. The patients were treated with DENO from January 2014 to December 2016, Zol from January 2017 to February 2019, and ROMO from March to December 2019, unless the patients hoped the other therapy. The switch from BPs to the abovementioned treatments showed no increase in BMD. Patients (1) treated with Zol (Zol group); (2) switched from selective estrogen receptor modulator, teriparatide, and DENO (ROMO group); (3) with a history of angina, cerebral, or myocardial infarction; (4) $<50$ years old; (5) who were male; and (6) with severe chronic kidney disease (stage 4 and 5), and (7) with abnormal serum levels of albumin-corrected calcium (Ca) $(<8.3$ or $>10.3 \mathrm{mg} /$ $\mathrm{dL})$ at baseline, were excluded from the study. Ten of the 112 patients treated with ROMO from March to December 2019 dropped out before 12 months -5 due to infection with the novel coronavirus, 1 moved cities, 1 suffered a cancer recurrence and switched to DENO treatment, and the other 3 due to unknown reasons. In this current study, we retrospectively investigated 73 patients treated with DENO from January 2014 to December 2016 and 81 patients treated with ROMO from March to December 2019. The following baseline clinical information was obtained: the levels of albumincorrected $\mathrm{Ca}$, bone metabolic markers, 25-dihydroxy vitamin D $[25(\mathrm{OH}) \mathrm{D}]$, intact parathyroid hormone $(\mathrm{PTH})$, and liver and renal function from the analysis of blood samples. BMD was evaluated using dual-energy X-ray absorptiometry (DXA); X-rays of the whole spine and full length of the lower limbs were obtained for evaluating previous fragility fractures and risk factors for atypical femoral fractures. The levels of serum albumin-adjusted calcium $(\mathrm{Ca})$, total type I procollagen $\mathrm{N}$-propeptide (P1NP), and tartrate-resistant acid

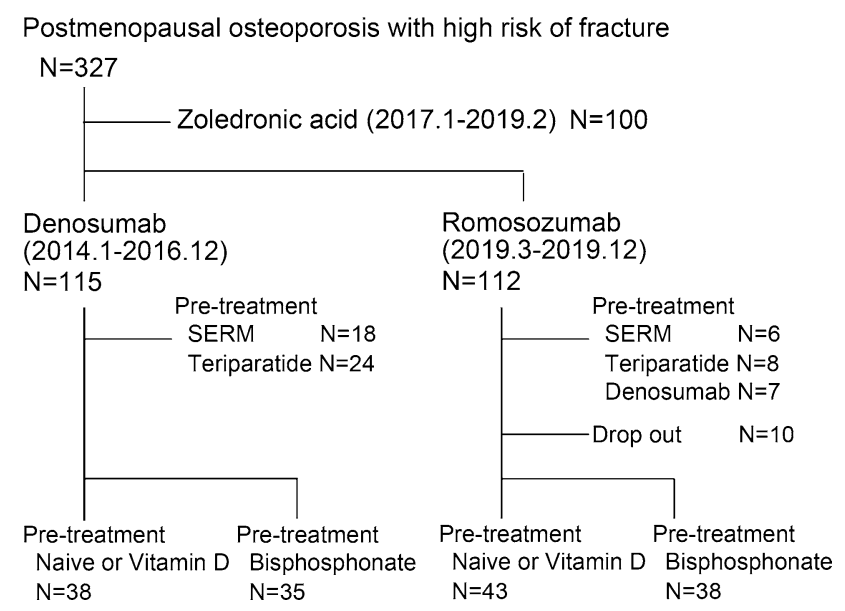

Fig. 1 Study design. SERM: selective estrogen receptor modulator 
phosphatase $5 b$ (TRACP $5 b$ ) were monitored at 3,6 , and 12 months after starting or switching to ROMO. Monitoring of serum $\mathrm{Ca}$ and inflammatory reactions was started after 1 or 2 weeks of DENO and ROMO administration to evaluate changes in $\mathrm{Ca}$ levels and assess side effects.

\section{BMD assessment}

Areal BMD of the lumbar spine (LS; L2-L4), femoral neck $(\mathrm{FN})$, and total hip (TH) was assessed at baseline and 6 and 12 months after treatment, using Horizon ${ }^{\circledR}$ DXA Bone Densitometer (Hologic Discovery A, Inc., Tokyo, Japan). Regions of severe scoliosis, previous vertebral fracture, and postoperative sites were excluded from BMD measurements; at least two of the L2-L4 lumbar vertebrae had to be evaluated for BMD [19]. Subjects were excluded from the BMD assessment if the area was fractured or operated on during the study.

\section{Statistical analysis}

Statistical comparisons between the groups were performed using unpaired $t$-test (durations of pretreatment), or two-way analysis of variance with the Bonferroni test for post hoc comparisons. Spearman's correlation coefficients were calculated, and linear regression models adjusted for age, body mass index (BMI), pretreatment with BPs and active vitamin $\mathrm{D}$, history of fragility fractures, and $25(\mathrm{OH}) \mathrm{D}$ levels were established to determine the associations with \% changes in BMD between the baseline and 12 months. All statistical analyses were performed using the Statistical Package for the Social Sciences (SPSS Statistics version 23.0) (IBM Corporation, Armonk, NY, USA) with the significance level set at 0.05 .

\section{Results}

\section{Clinical characteristics}

Table 1 shows the baseline characteristics. The number of patients who switched from naïve or vitamin $\mathrm{D}$ to ROMO (ND-ROMO), BPs to ROMO (BP-ROMO), ND to DENO (ND-DENO), and BPs to DENO (BP-DENO) was 43, 38, 38 , and 35, respectively. Two-way ANOVA showed statistically significant differences in mean BMI values between treatments (ROMO vs DENO) $(P=0.016)$ and pretreatments (ND vs BP) $(P=0.031)$. No interaction was detected between pretreatment and treatment values $(P=0.173)$. Post hoc Bonferroni tests showed that the mean BMI of patients was significantly higher in the ND-ROMO group than in the BP-ROMO group $(P=0.019)$. The mean duration of pretreatment with BP-DENO was significantly higher than that of pretreatment with BP-ROMO $(P=0.015)$. A total of 21 patients in the ND-ROMO group, 24 in the BP-ROMO group, 10 in the ND-DENO group, and 23 in the BP-DENO
Table 1 Clinical characteristics at baseline

\begin{tabular}{|c|c|c|c|c|}
\hline \multirow[b]{2}{*}{ Variable } & \multicolumn{2}{|c|}{ Romosozumab } & \multicolumn{2}{|l|}{ Denosumab } \\
\hline & ND & BPs & ND & BPs \\
\hline Number & 43 & 38 & 38 & 35 \\
\hline Age, years & $75.3(1.2)$ & $73.7(1.7)$ & $71.8(1.4)$ & $74.4(1.5)$ \\
\hline $\mathrm{BMI}^{*}, \dagger, \mathrm{kg} / \mathrm{m}^{2}$ & $22.1(0.6)$ & $20.0(0.6)$ & $22.7(0.5)$ & $22.2(0.6)$ \\
\hline Duration of pretreatment ${ }^{*}$, months & NA & $26.2(3.9)$ & NA & $43.8(5.9)$ \\
\hline Prior active vitamin D3 use & 21 patients & 24 patients & 10 patients & 23 patients \\
\hline History of fragility fractures & 34 patients & 22 patients & 21 patients & 23 patients \\
\hline $\mathrm{Ca}, \mathrm{mg} / \mathrm{dL}$ & $9.51(0.08)$ & $9.56(0.09)$ & $9.47(0.06)$ & $9.58(0.07)$ \\
\hline $\mathrm{eGFR}, \mathrm{mL} / \mathrm{min} / 1.73 \mathrm{~mm}^{2}$ & $65.8(2.4)$ & $68.4(2.7)$ & $73.4(2.3)$ & $69.2(3.2)$ \\
\hline 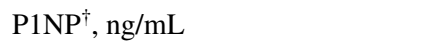 & $62.7(5.9)$ & $30.2(4.6)$ & $56.3(5.4)$ & $21.6(2.0)$ \\
\hline TRACP-5 $\mathrm{b}^{\dagger}, \mathrm{mU} / \mathrm{dL}$ & $497.6(37.2)$ & $285.5(28.5)$ & $451.0(27.2)$ & $279.5(20.2)$ \\
\hline $25(\mathrm{OH}) \mathrm{D}, \mathrm{ng} / \mathrm{mL}$ & $15.3(0.8)$ & $17.5(0.9)$ & $17.7(1.0)$ & $17.0(1.2)$ \\
\hline Intact PTH, pg/mL & $42.2(3.4)$ & $32.8(4.1)$ & $37.1(1.1)$ & $36.8(2.4)$ \\
\hline$\%$ YAM Lumbar, \% & $71.5(1.9)$ & $68.2(2.7)$ & $68.5(2.1)$ & $71.7(2.1)$ \\
\hline$\%$ YAM FN, $\%$ & $62.5(2.0)$ & $62.1(1.6)$ & $61.8(1.6)$ & $62.2(1.6)$ \\
\hline \% YAM ТН, \% & $69.6(2.2)$ & $67.5(1.8)$ & $68.0(1.7)$ & $65.9(1.9)$ \\
\hline
\end{tabular}

Mean (standard error of the mean). *: $P<0.05$ romosozumab vs. denosumab, $\uparrow: P<0.05$ ND vs. BP group $N D$ naïve or vitamin D, BPs bisphosphonates, $B M I$ body mass index, $C a$ calcium, $C r$ creatinine, $e G F R$ estimated glomerular filtration rate, $P I N P$ total type 1 procollagen-N-propeptide, $T R A C P-5 b$ tartrate-resistant acid phosphatase $5 \mathrm{~b}, 25(\mathrm{OH}) \mathrm{D} 25$-hydroxy vitamin $\mathrm{D}, P T H$ parathyroid hormone, YAM young adult mean, $F N$ femoral neck, $T H$ total hip 
group used active vitamin D3. The number of patients who experienced fragility fractures was $34,22,21$, and 23 in the ND-ROMO, BP-ROMO, ND-DENO, and BP-DENO groups, respectively. Two-way ANOVA showed a statistical difference between pretreatments (ND vs BP) in the mean of bone metabolic markers (P1NP: $P<0.001$ and TRACP-5b: $P<0.001)$. No interaction was detected between pretreatment and treatment values (P1NP: $P=0.994$ and TRACP5b: $P=0.918$ ). Post hoc Bonferroni tests showed that the mean of bone metabolic markers of patients in the NDROMO group was significantly higher than that of those in the BP-ROMO group (P1NP: $P<0.001$ and TRACP-5b: $P<0.001)$ and that of patients in the ND-DENO group was significantly higher than that of those in the BP-DENO group (P1NP: $P<0.001$ and TRACP-5b: $P<0.001$ ). There were no differences in age, $\mathrm{Ca}$ levels, estimated glemerular filtration rate (eGFR) levels, 25(OH)D levels, intact PTH level, and percent young adult mean (\% YAM) at baseline, between the groups. Notably, one patient each in the BPROMO experienced the right calcaneus fracture at 6 months after administration. One paint in the ND-DENO group and two patients in the BP-DENO group were observed the occurrence of new vertebral body fracture at 12 months after administration.

\section{Longitudinal changes in Ca, P1NP, and TRACP 5b levels}

For the decrease in serum Ca levels from baseline to 1 or 2 weeks, two-way ANOVA showed a statistically significant difference between pretreatments (ND vs BP) $(P=0.031)$. No interaction was detected between the pretreatment and treatment values $(P=0.485)$. Post hoc Bonferroni tests did not show significant differences between ND and BP in the ROMO and DENO groups (ROMO: $P=0.491$ and DENO: $P=0.093)$. While no patients showed hypocalcaemia (Ca level <8.4) in the ROMO group, three patients in the NDROMO group showed hypocalcaemia. However, none experienced serious side effects related to it (tonic convulsions and tetany).

For the change of P1NP levels at 3, 6, and 12 months, two-way ANOVA showed statistically significant differences between pretreatments (ND vs BP) ( 3 months: $P=0.003$, 6 months: $P<0.001$, and 12 months: $P<0.001)$ and treatments (ROMO vs DMAb) (3 months: $P<0.001,6$ months: $P<0.001$, and 12 months: $P<0.001$ ) (Fig. 2a). There were significant interactions between pretreatment and treatment values at 6 and 12 months ( 6 months: $P=0.026$ and 12 months: $P=0.009)$. For the change of TRACP-5b levels at 3, 6, and 12 months, two-way ANOVA showed statistically significant differences between pretreatments (ND vs BP) $(3$ months: $P<0.003,6$ months: $P=0.001$, and 12 months: $P<0.001)$ and treatments (ROMO vs DENO) (3 months: $P<0.001,6$ months: $P<0.001$, and 12 months: $P<0.001$ ) (Fig. 2b). No interaction was detected between the pretreatment and treatment values.

\section{Longitudinal changes in BMD}

At 6 and 12 months after the administration of ROMO or DENO, all groups showed increases in lumbar spine, femoral neck, and total hip BMDs compared to those at baseline (Fig. 3). For the change in BMD of the lumbar spine at 6 and 12 months, two-way ANOVA showed a statistically significant difference between treatments (ROMO vs DENO) (6 months: $P<0.001$ and 12 months: $P<0.001$ ) (Fig. 3a). No interaction was detected between the pretreatment and treatment values. For the change in BMD of the femoral neck at 6 months, two-way ANOVA showed a statistically significant difference between pretreatments (ND vs BP) $(P=0.031)($ Fig. $3 b)$. For that at 12 months, two-way ANOVA showed a statistically significant difference between
Fig. 2 Comparison of longitudinal changes in a serum P1NP and $\mathbf{b}$ serum TRACP-5b levels at 3-, 6-, and 12-month post-administration. Data show mean \pm standard error of the mean, $* P<0.05$ : Romosozumab vs. Denosumab, $\dagger P<0.05$ : nonbisphosphonate vs. bisphosphonate. $\# P<0.05$, interaction. $B P$ bisphosphonates, $P 1 N P$ total type 1 procollagen- $\mathrm{N}$-propeptide, TRACP-5b tartrate-resistant acid phosphatase $5 \mathrm{~b}$ a

- Romosozumab switched from naîve or vitamin $\mathrm{D}(\mathrm{N}=43)$

- Romosozumab switched from $B P(N=38)$

Denosumab switched from naive or vitamin $\mathrm{D}(\mathrm{N}=38)$

$\square$ Denosumab switched from BP ( $N=35)$

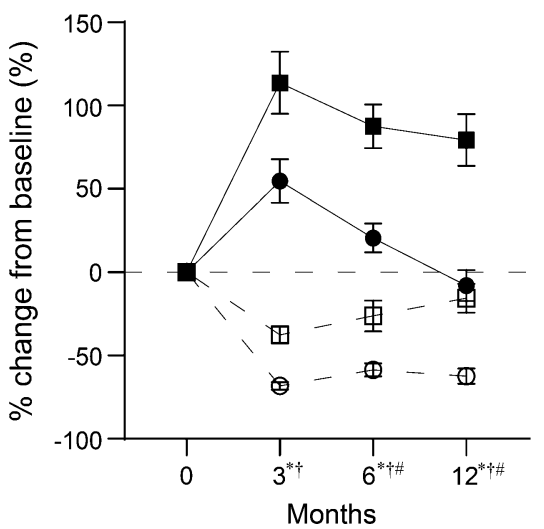

b

- Romosozumab switched from naïve or vitamin $D(N=43)$

Romosozumab switched from $\mathrm{BP}(\mathrm{N}=38)$

Denosumab switched from naïve or vitamin $D(N=38)$

Denosumab switched from BP $(\mathrm{N}=35)$

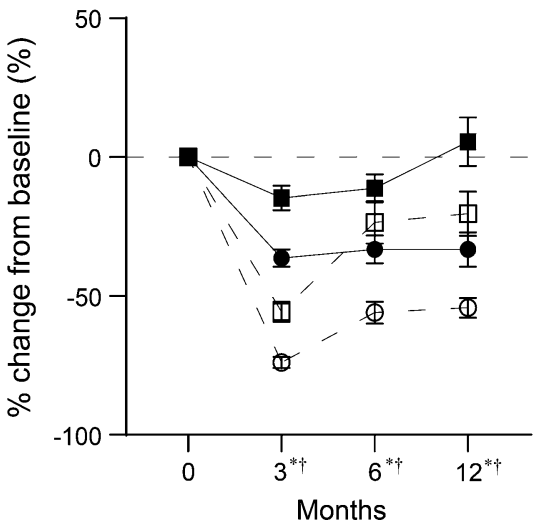



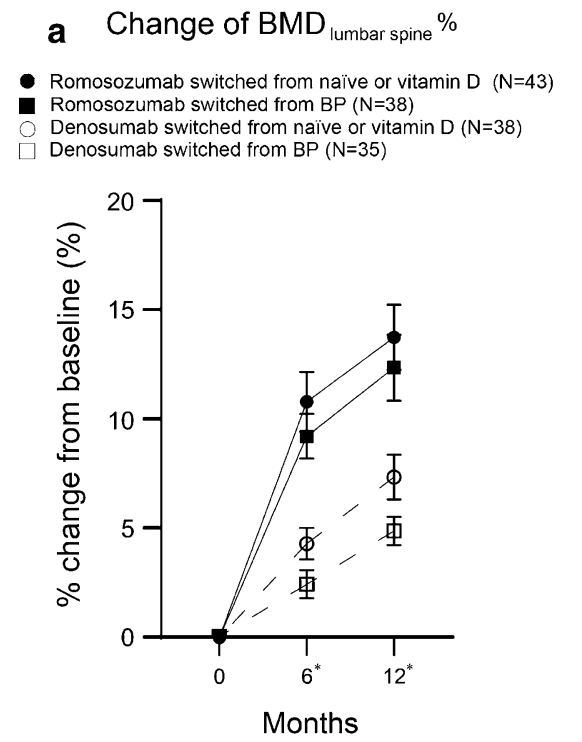
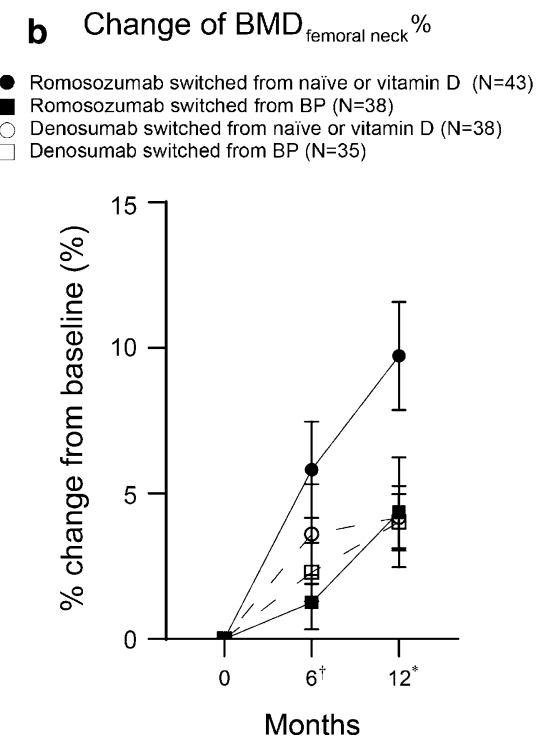

c Change of $B M D_{\text {total hip } \%}$

Romosozumab switched from naive or vitamin $D(N=43)$ Romosozumab switched from $\mathrm{BP}(\mathrm{N}=38)$ Denosumab switched from naïve or vitamin $\mathrm{D}(\mathrm{N}=38)$ Denosumab switched from $\mathrm{BP}(\mathrm{N}=35)$

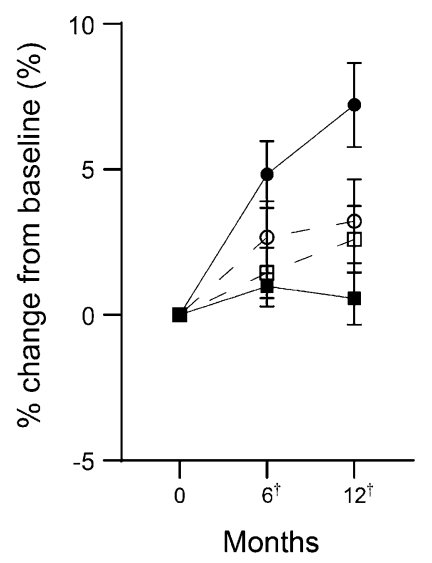

Fig. 3 Longitudinal \% changes in bone mineral densities of the a lumbar, b femoral neck, and $\mathbf{c}$ total hip. Data show mean \pm standard error of the mean. ${ }^{*} P<0.05$ : Romosozumab vs. Denosumab, $\dagger P<0.05$ : non-bisphosphonate vs. bisphosphonate. $B P$ bisphosphonate, $B M D$ bone mineral density

In the linear regression models adjusted for age, body mass index (BMI), pretreatment with BPs and active vitamin $\mathrm{D}$, history of fragility fractures, and $25(\mathrm{OH}) \mathrm{D}$ levels, the $\%$ changes of BMD of the lumber spine from baseline to 12 months were associated with P1NP value at baseline ( $\beta=0.439, P=0.003)$, and TRACP-5b value at baseline $(\beta=0.576, P<0.001)$ and its percentage change at 3 months ( $\beta=-0.528, P=0.003$ ) (Table 2). Percentage changes of BMD of the femoral neck from baseline to 12 months were associated with $\mathrm{P} 1 \mathrm{NP}(\beta=0.343, P=0.049)$ and TRACP-5b $(\beta=0.467, P=0.015)$ values at baseline. Percentage changes of BMD of the total hip from baseline to 12 months were associated with $\mathrm{P} 1 \mathrm{NP}$ value at baseline ( $\beta=0.434, P=0.003)$, and TRACP-5b value at baseline $(\beta=0.329, P=0.037)$ and its percentage change at 3 months $(\beta=-0.407, P=0.029)$.

\section{Discussion}

This study investigated the longitudinal efficacy of ROMO over 12 months, the maximum period approved for insurance practice in Japan, in a real-world setting. It showed that ROMO increased the BMDs of the lumbar spine, femoral neck, and total hip at 12 months. This finding was consistent with that of a previous clinical trial [20-22] and a 6-month observational study in a real-world setting [11, 12] on Japanese patients with postmenopausal osteoporosis. Considering that the ND-ROMO and BP-ROMO groups showed increases in BMDs of the lumbar spine, femoral neck, and total hip from 6 to 12 months regardless of the decrease in 
Table 2 Linear regression models of association of changes in bone mineral density among patients treated by Romosozumab

\begin{tabular}{|c|c|c|c|c|}
\hline Factor & $\beta$ & \multicolumn{2}{|c|}{ 95\% Confidence interval } & $P$ value \\
\hline \multicolumn{5}{|c|}{$\%$ Change of BMD of the lumbar spine } \\
\hline P1NP at BL & 0.439 & 0.029 & 0.134 & 0.003 \\
\hline TRACP-5b at BL & 0.576 & 0.010 & 0.029 & $<0.001$ \\
\hline$\%$ Change of P1NP at $3 \mathrm{M}$ & -0.136 & -0.033 & 0.014 & 0.422 \\
\hline$\%$ Change of TRACP-5b at $3 \mathrm{M}$ & -0.528 & -0.213 & -0.047 & 0.003 \\
\hline \multicolumn{5}{|c|}{$\%$ Change of BMD of the femoral neck } \\
\hline P1NP at BL & 0.343 & 0.001 & 0.209 & 0.049 \\
\hline TRACP-5b at BL & 0.467 & 0.006 & 0.048 & 0.015 \\
\hline$\%$ Change of P1NP at $3 \mathrm{M}$ & 0.090 & -0.030 & 0.050 & 0.621 \\
\hline$\%$ Change of TRACP-5b at $3 \mathrm{M}$ & -0.160 & -0.216 & 0.093 & 0.424 \\
\hline \multicolumn{5}{|l|}{$\%$ Change of BMD of the total hip } \\
\hline P1NP at BL & 0.434 & 0.029 & 0.139 & 0.003 \\
\hline TRACP-5b at BL & 0.329 & 0.008 & 0.024 & 0.037 \\
\hline$\%$ Change of P1NP at $3 \mathrm{M}$ & -0.079 & -0.030 & 0.019 & 0.659 \\
\hline$\%$ Change of TRACP-5b at $3 \mathrm{M}$ & -0.407 & -0.190 & -0.011 & 0.029 \\
\hline
\end{tabular}

Adjusted by age, body mass index, pretreatment with BPs and active vitamin D, history of fragility fractures, and 25(OH)D levels

$P 1 N P$ total type 1 procollagen-N-propeptide, TRACP-5b tartrate-resistant acid phosphatase $5 \mathrm{~b}, 25(O H) D$ 25-hydroxy vitamin $\mathrm{D}, B L$ baseline, $3 M 3$ months dual effect, increase in bone formation, and decrease in bone resorption [4, 5], continuous administration of ROMO for 12 months could potentially increase BMD.

Consistent with previous reports [11,23], we found that the bone metabolic markers at baseline were associated with changes in BMD over 12 months in patients treated with ROMO. Therefore, bone metabolic markers at baseline are useful predictors of BMD during ROMO treatment. This finding suggested that pretreatment with bisphosphonate decreased the increase of BMD with ROMO. Contrary to other clinical observations [12], this study did not show any association between early changes in P1NP and changes in BMD at 12 months. However, we observed that early changes in TRACP-5b were associated with changes in BMD at 12 months. This discrepancy may be attributed to the current study evaluating only the 3 months change and not the 1 month change as previously described [12]. Considering that ROMO was associated with a transient increase in bone formation markers and sustained decrease in boneresorption markers [5], earlier changes in bone metabolic markers, such as in 1 month, might be more appropriate for predicting changes in BMD.

This study showed that ROMO significantly increased the lumbar spine and femoral neck BMD at 12 months compared to DENO. Therefore, ROMO is more effective in increasing BMD than DENO, alendronate, and teriparatide; this finding is in line with results shown in a phase 2, multicentre, international, and randomized control study [5]. On the other hand, this study also showed that the effects of ROMO switched from BP on BMD of femoral neck and total hip were almost same with DENO. Therefore, 12-month ROMO treatment may not be a more ideal and sufficient treatment for patients with low BMD of femoral neck or total hip who received BP therapy previously. To the best of our knowledge, this is the first study that compares the efficacy of ROMO and DENO in postmenopausal osteoporosis patients with a high risk of fracture. Because the characteristics of patients recruited varied with time, the comparison between ROMO and DENO may not be accurate; this is the main limitation of this study. Therefore, a randomized control comparison study is warranted in the future to confirm the superiority of ROMO over DENO.

The FRAME trial showed that BMD continued to increase after switching from ROMO to DENO [7], and Ebina et al. showed that pretreatment for bone resorption attenuated the increase of BMD in ROMO compared to that in the control group [11]. Additionally, Kashii et al. showed that ROMO is not effective in preventing vertebral fractures or multiple spontaneous clinical vertebral fractures after DENO discontinuation [24]. Therefore, it might be better for postmenopausal patients with a high risk of fracture to be treated first with ROMO rather than with bone resorption inhibitors. Comparison studies in the future should address this speculation to establish a therapeutic strategy for osteoporosis with a high risk of fracture.

This study has some limitations. First, the study had a small sample size. Therefore, the statistical power of the results might be attenuated, and the fracture occurrence rate after initiation of treatment might be underestimated. Second, this study included pretreatment with various BPs, 
including alendronate and risedronate, and active vitamin D3, such as alfacalcidol and ELD, which might have affected the results. Because this was not a randomized study, differences in patients' backgrounds may potentially affect the physicians' treatment selection and subsequent effects. Larger randomized studies with longer follow-up periods should be conducted in the future.

In conclusion, in this 12-month follow-up study of postmenopausal osteoporosis patients with a high risk of fracture who were introduced to ROMO or DENO in a real-world setting, ROMO continuously increased BMD for 12 months and performed better than DENO. On the other hand, effects of ROMO switched from BP on BMD of femoral neck and total hip were almost same with DENO. For patients treated by ROMO, bone metabolic markers at baseline and changes in TRACP-5b levels from the baseline to 3 months were associated with changes in BMD from baseline to 12 months. These findings suggest that these parameters could be useful in predicting the efficacy of ROMO. Although these results may contribute to establishing a therapeutic strategy for osteoporosis with a high risk of fracture, further investigations are required.

Acknowledgements This study was funded by Grant-in-Aid for Young Scientists from the Ministry of Education, Culture, Sports, Science, and Technology of Japan 20K17948 (T. Shimizu).

\section{Declarations}

Conflict of interest Tomohiro Shimizu, Kosuke Arita, Eihiro Murota, Shigeto Hiratsuka, Ryo Fujita, Tsuyoshi Asano, Masahiko Takahata and Norimasa Iwasaki declare that they have no conflict of interest.

\section{References}

1. Johnell O, Kanis JA (2006) An estimate of the worldwide prevalence and disability associated with osteoporotic fractures. Osteoporos Int 17:1726-1733. https://doi.org/10.1007/ s00198-006-0172-4

2. EFFO N (1997) Who are candidates for prevention and treatment for osteoporosis? Osteoporos Int 7:1-6

3. Oden A, McCloskey EV, Kanis JA, Harvey NC, Johansson H (2015) Burden of high fracture probability worldwide: secular increases 2010-2040. Osteoporos Int 26:2243-2248. https://doi. org/10.1007/s00198-015-3154-6

4. Padhi D, Jang G, Stouch B, Fang L, Posvar E (2011) Single-dose, placebo-controlled, randomized study of AMG 785, a sclerostin monoclonal antibody. J Bone Miner Res 26:19-26. https://doi.org/ 10.1002/jbmr.173

5. McClung MR, Grauer A, Boonen S, Bolognese MA, Brown JP, Diez-Perez A, Langdahl BL, Reginster JY, Zanchetta JR, Wasserman SM, Katz L, Maddox J, Yang YC, Libanati C, Bone HG (2014) Romosozumab in postmenopausal women with low bone mineral density. N Engl J Med 370:412-420. https://doi.org/10. 1056/NEJMoa1305224

6. Gennari L, Rotatori S, Bianciardi S, Nuti R, Merlotti D (2016) Treatment needs and current options for postmenopausal osteoporosis. Expert Opin Pharmacother 17:1141-1152. https:// doi.org/10.1080/14656566.2016.1176147

7. Cosman F, Crittenden DB, Adachi JD, Binkley N, Czerwinski E, Ferrari S, Hofbauer LC, Lau E, Lewiecki EM, Miyauchi A, Zerbini CA, Milmont CE, Chen L, Maddox J, Meisner PD, Libanati C, Grauer A (2016) Romosozumab Treatment in Postmenopausal Women with Osteoporosis. N Engl J Med 375:1532-1543. https://doi.org/10.1056/NEJMoa1607948

8. Saag KG, Petersen J, Brandi ML, Karaplis AC, Lorentzon M, Thomas T, Maddox J, Fan M, Meisner PD, Grauer A (2017) Romosozumab or Alendronate for Fracture Prevention in Women with Osteoporosis. N Engl J Med 377:1417-1427. https://doi.org/ 10.1056/NEJMoa1708322

9. Langdahl BL, Libanati C, Crittenden DB, Bolognese MA, Brown JP et al (2017) Romosozumab (sclerostin monoclonal antibody) versus teriparatide in postmenopausal women with osteoporosis transitioning from oral bisphosphonate therapy: a randomised, open-label, phase 3 trial. Lancet 390:1585-1594. https://doi.org/ 10.1016/S0140-6736(17)31613-6

10. Lacey DL, Boyle WJ, Simonet WS, Kostenuik PJ, Dougall WC, Sullivan JK, San Martin J, Dansey R (2012) Bench to bedside: elucidation of the OPG-RANK-RANKL pathway and the development of denosumab. Nat Rev Drug Discov 11:401-419. https:// doi.org/10.1038/nrd3705

11. Ebina K, Hirao M, Tsuboi H, Nagayama Y, Kashii M, Kaneshiro S, Miyama A, Nakaya H, Kunugiza Y, Okamura G, Etani Y, Takami K, Goshima A, Nakata K (2020) Effects of prior osteoporosis treatment on early treatment response of romosozumab in patients with postmenopausal osteoporosis. Bone 140:115574. https://doi.org/10.1016/j.bone.2020.115574

12. Tominaga A, Wada K, Kato Y, Nishi H, Terayama Y, Okazaki K (2020) Early clinical effects, safety, and appropriate selection of bone markers in romosozumab treatment for osteoporosis patients: a 6-month study. Osteoporos Int. https://doi.org/10.1007/ s00198-020-05639-y

13. Orimo H, Nakamura T, Hosoi T, Iki M, Uenishi K, Endo N, Ohta H, Shiraki M, Sugimoto T, Suzuki T, Soen S, Nishizawa Y, Hagino H, Fukunaga M, Fujiwara S (2012) Japanese 2011 guidelines for prevention and treatment of osteoporosis-executive summary. Arch Osteoporos 7:3-20. https://doi.org/10.1007/ s11657-012-0109-9

14. Soen S, Fukunaga M, Sugimoto T, Sone T, Fujiwara S, Endo N, Gorai I, Shiraki M, Hagino H, Hosoi T, Ohta H, Yoneda T, Tomomitsu T, Japanese Society for B, Mineral R, Japan Osteoporosis Society Joint Review Committee for the Revision of the Diagnostic Criteria for Primary O (2013) Diagnostic criteria for primary osteoporosis: year 2012 revision. J Bone Miner Metab 31:247-257. https://doi.org/10.1007/s00774-013-0447-8

15. Marcus R, Wang O, Satterwhite J, Mitlak B (2003) The skeletal response to teriparatide is largely independent of age, initial bone mineral density, and prevalent vertebral fractures in postmenopausal women with osteoporosis. J Bone Miner Res 18:18-23. https:// doi.org/10.1359/jbmr.2003.18.1.18

16. Shiraki M, Kuroda T, Miyakawa N, Fujinawa N, Tanzawa K et al (2011) Design of a pragmatic approach to evaluate the effectiveness of concurrent treatment for the prevention of osteoporotic fractures: rationale, aims and organization of a Japanese Osteoporosis Intervention Trial (JOINT) initiated by the Research Group of Adequate Treatment of Osteoporosis (A-TOP). J Bone Miner Metab 29:37-43. https://doi.org/10.1007/s00774-010-0188-x

17. Gallagher JC, Genant HK, Crans GG, Vargas SJ, Krege JH (2005) Teriparatide reduces the fracture risk associated with increasing number and severity of osteoporotic fractures. J Clin Endocrinol Metab 90:1583-1587. https://doi.org/10.1210/jc.2004-0826

18. Genant HK, Wu CY, van Kuijk C, Nevitt MC (1993) Vertebral fracture assessment using a semiquantitative technique. J Bone 
Miner Res 8:1137-1148. https://doi.org/10.1002/jbmr.56500 80915

19. Ebina K, Hashimoto J, Shi K, Kashii M, Hirao M, Yoshikawa $\mathrm{H}$ (2015) Undercarboxylated osteocalcin may be an attractive marker of teriparatide treatment in RA patients: response to Mokuda. Osteoporos Int 26:1445. https://doi.org/10.1007/ s00198-014-2993-x

20. Ishibashi H, Crittenden DB, Miyauchi A, Libanati C, Maddox J, Fan M, Chen L, Grauer A (2017) Romosozumab increases bone mineral density in postmenopausal Japanese women with osteoporosis: a phase 2 study. Bone 103:209-215. https://doi.org/10. 1016/j.bone.2017.07.005

21. Miyauchi A, Dinavahi RV, Crittenden DB, Yang W, Maddox JC, Hamaya E, Nakamura Y, Libanati C, Grauer A, Shimauchi J (2019) Increased bone mineral density for 1 year of romosozumab, vs placebo, followed by 2 years of denosumab in the Japanese subgroup of the pivotal FRAME trial and extension. Arch Osteoporos 14:59. https://doi.org/10.1007/s11657-019-0608-z

22. Miyauchi A, Hamaya E, Yang W, Nishi K, Libanati C, Tolman C, Shimauchi J (2020) Romosozumab followed by denosumab in Japanese women with high fracture risk in the FRAME trial. J Bone Miner Metab. https://doi.org/10.1007/s00774-020-01147-5

23. Takada J, Dinavahi R, Miyauchi A, Hamaya E, Hirama T, Libanati C, Nakamura Y, Milmont CE, Grauer A (2020) Relationship between P1NP, a biochemical marker of bone turnover, and bone mineral density in patients transitioned from alendronate to romosozumab or teriparatide: a post hoc analysis of the STRUCTURE trial. J Bone Miner Metab 38:310-315. https://doi.org/10.1007/ s00774-019-01057-1

24. Kashii M, Ebina K, Kitaguchi K, Yoshikawa H (2020) Romosozumab was not effective in preventing multiple spontaneous clinical vertebral fractures after denosumab discontinuation: a case report. Bone Rep 13:100288. https://doi.org/10.1016/j.bonr. 2020.100288

Publisher's Note Springer Nature remains neutral with regard to jurisdictional claims in published maps and institutional affiliations. 